\title{
Neoadjuvant or adjuvant therapy for resectable gastric cancer: a systematic review and practice guideline for North America
}

\author{
Greg Knight $\cdot$ Craig C. Earle $\cdot$ Roxanne Cosby $\cdot$ \\ Natalie Coburn $\cdot$ Youssef Youssef $\cdot$ Richard Malthaner • \\ Rebecca K. S. Wong • The Gastrointestinal Cancer Disease Site Group
}

Received: 23 September 2011/ Accepted: 16 February 2012/Published online: 31 March 2012

(c) The International Gastric Cancer Association and The Japanese Gastric Cancer Association 2012

\begin{abstract}
Background Gastric cancer is a global health problem accounting for $10 \%$ of all new cancer cases and $12 \%$ of all cancer deaths worldwide. Many clinical trials and metaanalyses have explored the value of neoadjuvant or adjuvant chemotherapy and radiation therapy in gastric cancer; however, these studies have produced conflicting results. The purpose of this guidance document was to determine
\end{abstract}

Electronic supplementary material The online version of this article (doi:10.1007/s10120-012-0148-3) contains supplementary material, which is available to authorized users.

G. Knight $(\bowtie)$

Grand River Regional Cancer Centre, 835 King Street West,

P O Box 9056, Kitchener, ON N2G 1G3, Canada

e-mail: gregory.knight@grhosp.on.ca

C. C. Earle · N. Coburn

Odette Cancer Centre, Toronto, ON, Canada

R. Cosby

Program in Evidence-Based Care, McMaster University, Juravinski Site, G Wing, 2nd Floor, 711 Concession Street, Hamilton, ON L8V 1C3, Canada

e-mail: cosbyr@mcmaster.ca

R. Cosby

Department of Oncology, McMaster University,

Hamilton, ON, Canada

Y. Youssef

Durham Regional Cancer Centre, Oshawa, ON, Canada

R. Malthaner

London Health Sciences Centre, London, ON, Canada

R. K. S. Wong

Princess Margaret Hospital, Toronto, ON, Canada whether patients with resectable gastric cancer should receive neoadjuvant or adjuvant therapy in addition to surgery. Outcomes of interest were overall survival, disease-free survival, and adverse events.

Methods A systematic review was undertaken to inform recommendations regarding neoadjuvant and adjuvant therapy in resectable gastric cancer in Ontario, Canada. MEDLINE and EMBASE databases, as well as American Society of Clinical Oncology (ASCO) annual meeting proceedings and American Society for Therapeutic Radiology and Oncology (ASTRO) proceedings were systematically searched from 2002 to 2010. Oral fluoropyrimidine trials were excluded owing to the unavailability of these agents in North America.

Results Overall, 22 randomized controlled trials (RCTs), 13 meta-analyses, and two secondary analyses were included. The systematic review informed the development of a clinical practice guideline with the following recommendations. Postoperative 5-fluorouracil-based chemoradiotherapy based on the Macdonald approach or perioperative ECF (epirubicin, cisplatin, fluorouracil) chemotherapy based on the Cunningham/MAGIC (Medical Research Council Adjuvant Gastric Infusional Chemotherapy) approach are both acceptable standards of care in North America. Choice of treatment should be made on a case-by-case basis. Adjuvant chemotherapy is a reasonable option for those patients for whom the Macdonald and MAGIC protocols are contraindicated. All patients with resectable gastric cancer should undergo a pretreatment multidisciplinary assessment to determine the best plan of care.

Conclusions Overall survival in patients with resectable gastric cancer is significantly improved with the use of either postoperative chemoradiation (Macdonald approach) or perioperative ECF (MAGIC protocol). 
Keywords Adjuvant therapy - Clinical practice guideline - Gastric cancer · Neoadjuvant therapy $\cdot$ Systematic review

\begin{tabular}{|c|c|}
\hline \multicolumn{2}{|c|}{ Abbreviations } \\
\hline $5-\mathrm{FU}$ & 5-Fluorouracil \\
\hline EtLF & Etoposide leucovorin/folinic acid \\
\hline FAM & Fluorouracil adriamycin, mitomycin \\
\hline FM & Fluorouracil mitomycin \\
\hline Mf & Mitomycin C oral fluoropyrimidine (doxifluridine) \\
\hline MfP & $\begin{array}{l}\text { Mitomycin C oral fluoropyrimidine (doxifluridine), } \\
\text { cisplatin }\end{array}$ \\
\hline MMC & Mitomycin C \\
\hline PELF & Cisplatin epirubicin, leucovorin/folinic acid \\
\hline
\end{tabular}

\section{Introduction}

Although the incidence and mortality of gastric cancer have been steadily decreasing in Canadian men and women, this disease remains a global health problem, accounting for $10 \%$ of all new cancer cases and $12 \%$ of all cancer deaths worldwide [1]. In Canada, the annual percent changes in age-standardized incidence between 1996 and 2005 were -2.3 and $-1.9 \%$ in males and females, respectively. The corresponding numbers for the change in age-standardized mortality between 1995 and 2004 were -3.6 and $-3.1 \%$ for males and females, respectively [2]. In Ontario in 2009, there will be an estimated 1090 new incident cases of stomach cancer $(38 \%$ of new incident stomach cancer cases in Canada) and 670 deaths from stomach cancer (36\% of stomach cancer deaths in Canada). The 5-year relative survival ratio is $23 \%$ (95\% confidence interval [CI] 21-24\%) for males and females combined [2]. However, the 5-year survival rate is much higher (about $75 \%$ ) for patients with localized disease without regional lymph node involvement in whom the cancer is managed with surgery alone [3]. Because the prognosis worsens with progressive lymph node involvement, there is interest in finding ways to improve the treatment results for this group of patients.

Although many clinical trials and meta-analyses have explored the value of neoadjuvant or adjuvant chemotherapy and radiation therapy in gastric cancer, these studies have produced conflicting results [4-6], making the role of which neoadjuvant and adjuvant therapy to recommend controversial. Results of gastric cancer treatment have tended to be better for studies carried out in Asian countries, possibly because of etiologic or biologic differences in the disease or different practices such as screening for early-stage cancer, the use of extended lymph node dissection, and the commencement of chemotherapy immediately after surgery. This makes it difficult to extrapolate findings beyond Asia. The purpose of this guideline is to determine whether or not resectable gastric cancer [stage 1B (invasion of the muscularis propria) and above] patients in North America should receive neoadjuvant or adjuvant therapy in addition to surgery. Oral fluoropyrimidines will not be considered as they are unavailable in North America. Outcomes of interest are overall survival (OS), diseasefree survival (DFS), and adverse events.

This guideline is an update of the Program in EvidenceBased Care's Evidence-Based Series (EBS) \#2-14, which was originally developed in 2000 and then updated in 2003. In the previous version, adjuvant chemoradiation was recommended, and if a patient was unable to undergo radiation adjuvant chemotherapy was the alternative. The Gastrointestinal Disease Site Group (GI DSG) believed that this further update was warranted, given the existence of new evidence published that could change the recommendations provided in the previous guideline.

\section{Methods}

The EBS guidelines developed by Cancer Care Ontario's Program in Evidence-Based Care (PEBC) use the methods of the Practice Guidelines Development Cycle [7]. For this project, the core methodology used to develop the evidentiary base was the systematic review. Evidence was selected and reviewed by one member of the PEBC Gastrointestinal DSG and a methodologist.

The systematic review is a convenient and up-to-date source of the best available evidence on neoadjuvant or adjuvant therapy for resectable gastric cancer. The body of evidence in this review is primarily comprised of mature randomized controlled trial (RCT) data and meta-analyses of RCTs. That evidence forms the basis of the recommendations developed by the Gastrointestinal DSG. The systematic review and companion recommendations are intended to promote evidence-based practice in Ontario, Canada. The PEBC is supported by the Ontario Ministry of Health and Long-Term Care through Cancer Care Ontario. All work produced by the PEBC is editorially independent from its funding source.

\section{Literature search strategy}

The MEDLINE (January 2002 to June week 3 2010), EMBASE (2002-2010 week 25), and Cochrane Library (February 2010), databases were systematically searched. Literature search strategies are available in the supplementary material (Appendix 1). The proceedings of the 2002-2010 American Society of Clinical Oncology (ASCO) and the 2002-2009 American Society for Therapeutic Radiology and Oncology (ASTRO) annual meetings 
were also searched for abstract reports of relevant studies. Reference lists of relevant reviews were searched for additional relevant reports.

\section{Study selection criteria}

Articles were selected for inclusion if: (1) they were published abstracts of final data or fully published reports of RCTs comparing preoperative or postoperative chemotherapy and/or radiotherapy versus potentially curative surgery alone or another preoperative or postoperative therapy approach. Syntheses of RCTs in the form of systematic reviews or meta-analyses were also included; (2) they were studies of adults with resectable gastric cancer. Trials of gastric cancer that also included patients with tumors of the gastroesophageal junction (GEJ) were included; (3) they included reports of OS data; (4) they were published in English(owing to the unavailability of translation services).

\section{Study quality appraisal}

The quality of the systematic reviews and meta-analyses was assessed using the Assessment of Multiple Systematic Reviews (AMSTAR) tool [8]. Randomized trials were assessed for key methodological characteristics, using information provided in the trial reports. The following elements were assessed: generation of allocation sequence, allocation concealment, blinding, intention-to-treat analysis, withdrawals, loss to follow up, funding source, statistical power calculations, length of follow up, differences in baseline patient characteristics, and early termination (see Supplementary Materials, Appendix 2).

\section{Results}

Literature search results

The updated search of MEDLINE and EMBASE yielded 1129 articles, of which 149 were retrieved for full-text review following title and abstract screening. One hundred nineteen of the 149 articles were subsequently excluded because they were either duplicate citations or did not meet the inclusion criteria. One further meta-analysis conducted in Japan and that only included oral fluoropyrimidine trials was also excluded [9], owing to the unavailability of these agents in North America. Thirty-three abstracts from the ASCO annual meeting proceedings and six abstracts from the ASTRO proceedings were retrieved for review; 14 initially met the inclusion criteria. However, five of these 14 abstracts were reports of RCTs or meta-analyses that were subsequently fully published, and are not discussed further.
Two were meta-analyses conducted in Japan and only included studies of oral fluoropyrimidines, and they are not discussed further [10,11]. No additional relevant studies were identified in a search of the Cochrane Library. Overall, 22 RCTs [12-33], 13 meta-analyses [34-46], and two secondary analyses that report survival data $[47,48]$ are included in this systematic review (see Fig. 1). One article reported the results of two RCTs [17]. Six systematic reviews without meta-analyses were identified, but none were included in this report as meta-analytic data were available.

\section{Postoperative chemotherapy}

Seven published literature meta-analyses were identified that compared postoperative chemotherapy versus surgery alone for patients with resected gastric cancer [34-40]. Study inclusion criteria, literature search periods, and statistical methods differed between the seven meta-analyses, although the basic research question was the same. There was considerable overlap in the studies included in each of these meta-analyses. These seven meta-analyses will not be discussed further, owing to the availability of a recent individual patient data (IPD) meta-analysis [41]. These authors [41] identified 31 eligible trials from 1970 to 2009 and were able to obtain IPD from 17 of them. An examination of the eligible studies does not indicate any bias with respect to studies for which the authors were and were not able to obtain the IPD. These authors [41] used a fixedeffects model and determined that there was a modest advantage of postoperative chemotherapy for OS (hazard ratio [HR] $0.82,95 \%$ CI $0.76-0.90 ; p<0.001$ ) based on 17 trials, and for DFS (HR 0.82, 95\% CI 0.75-0.90; $p<0.001$ ) based on 14 trials. No heterogeneity was detected for either outcome measure. The GASTRIC group [41] subsequently conducted a sensitivity analysis for OS using IPD where available (17 trials) and published summary statistics for the other studies, where available (11 trials). The results of the sensitivity analysis were consistent with the main analysis for OS (HR 0.82, 95\% CI 0.77-0.88; $p<0.001$ ).

The GASTRIC group report [41] does not include information about adverse events. However, searching through the individual studies demonstrates that the most common grade 3 and 4 hematologic toxicities are leucopenia, thrombocytopenia, and neutropenia, depending on the chemotherapy regimen. The most common grade 3 and 4 non-hematologic toxicities, other than alopecia, are nausea and/or vomiting, diarrhea, mucositis, and stomatitis, depending on the chemotherapy regimen. Not all of the studies reported toxicity or graded the toxicity if they did report it; this was especially apparent in the older trials.

The literature search identified 11 trial reports, representing 12 RCTs that compared postoperative chemotherapy with surgery alone [12-22]. All of these studies, except 
Fig. 1 Literature search results. $A S C O$ American Society of Clinical Oncology, ASTRO American Society for Therapeutic Radiology and Oncology

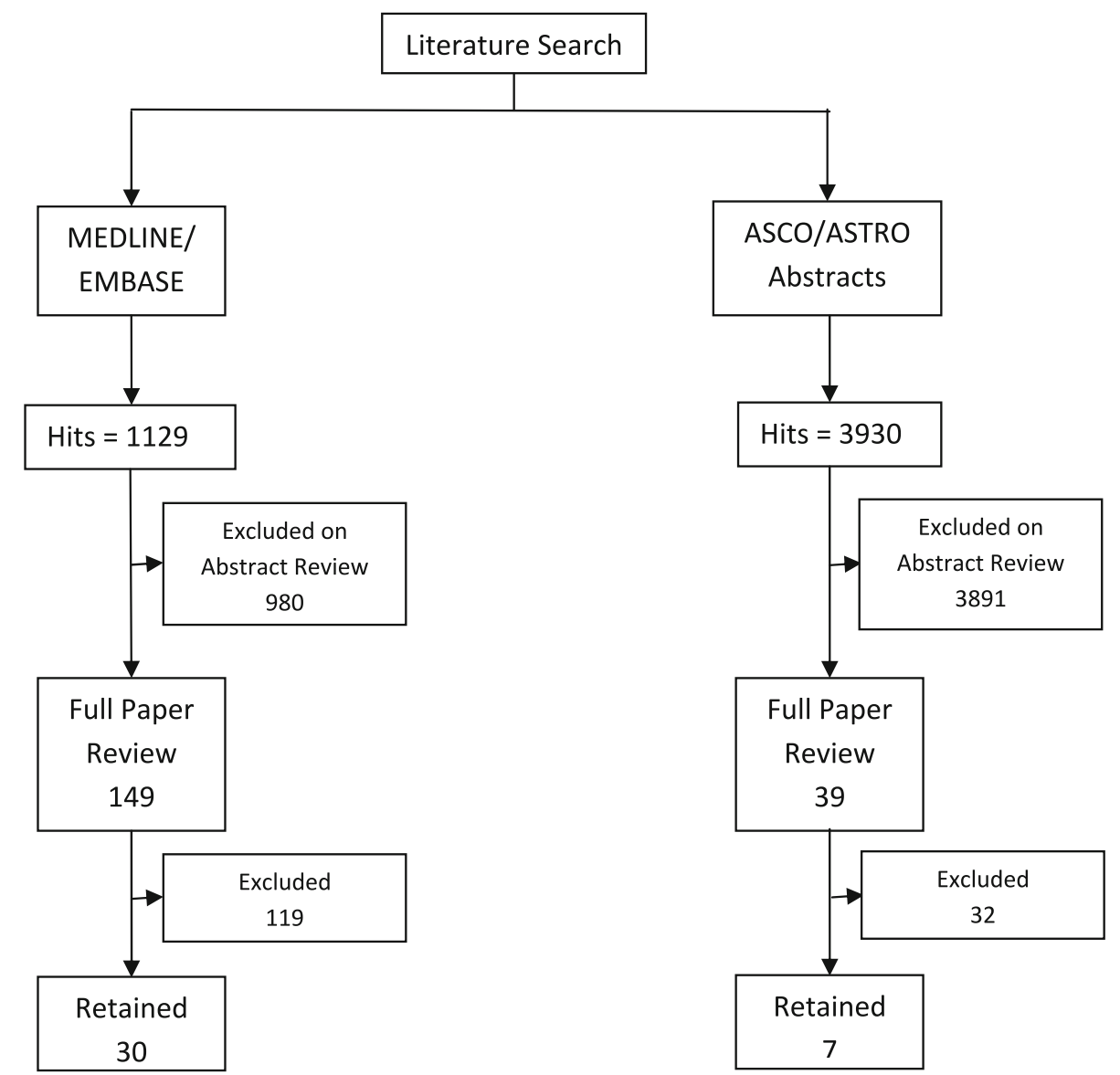

for one very recently published trial [22], were part of the meta-analyses described above and will not be discussed further. Kulig et al. [22] compared postoperative chemotherapy (etoposide, adriamycin, and cisplatin) to surgery alone. They reported no survival advantage in the chemotherapy arm. Grade 3 or 4 toxicities were reported in $22 \%$ of patients, with leucopenia being the most common toxicity reported $(6 \%)$.

Five RCTs compared postoperative chemotherapy versus another postoperative chemotherapy regimen [23-27]. These study results are summarized in Table 1. Three trials did not demonstrate a difference in OS, DFS, or local recurrence between treatment arms: one compared fluorouracil adriamycin, mitomycin (FAM) versus fluorouracil mitomycin (FM) versus 5-fluorouracil (5-FU) [23]; one compared cisplatin epirubicin, leucovorin/folinic acid (PELF) versus 5-FU [25]; and one compared mitomycin C oral fluoropyrimidine (doxifluridine), cisplatin (MfP) versus mitomycin $\mathrm{C}$ oral fluoropyrimidine (doxifluridine) (Mf) [27]. A small trial comparing PELF versus etoposide leucovorin/folinic acid (EtLF) for completely resected advanced gastric cancer (clinical stage 3 or 4, M0) reported a significant benefit for PELF in OS and DFS [24]. Another trial comparing folinic acid (leucovorin), flurouracil, irinotecan (FOLFIRI)/docetaxel/cisplatin versus mitomycin C (MMC) was stopped early for evidence of a DFS benefit favoring FOLFIRI/docetaxel/cisplatin at an unplanned interim analysis. Therefore, the results should be interpreted with caution [26]. Other than alopecia, hematologic toxicities (leucopenia, thrombocytopenia, and neutropenia) and nausea and vomiting were the most often reported grade 3 and 4 toxicities, especially for regimens involving cisplatin, etoposide, or epirubicin.

Postoperative radiotherapy

No meta-analyses or RCTs solely comparing postoperative radiotherapy versus surgery alone for resectable gastric cancer were identified in the updated literature search.

\section{Postoperative chemoradiotherapy (CRT)}

One Phase III RCT comparing postoperative CRT versus postoperative chemotherapy was identified [28]. Initially, the chemotherapy regimen consisted of docetaxel and cisplatin. However, the cisplatin was subsequently changed to carboplatin, owing to high rates of nausea and vomiting. The arms did not differ significantly with respect to median 


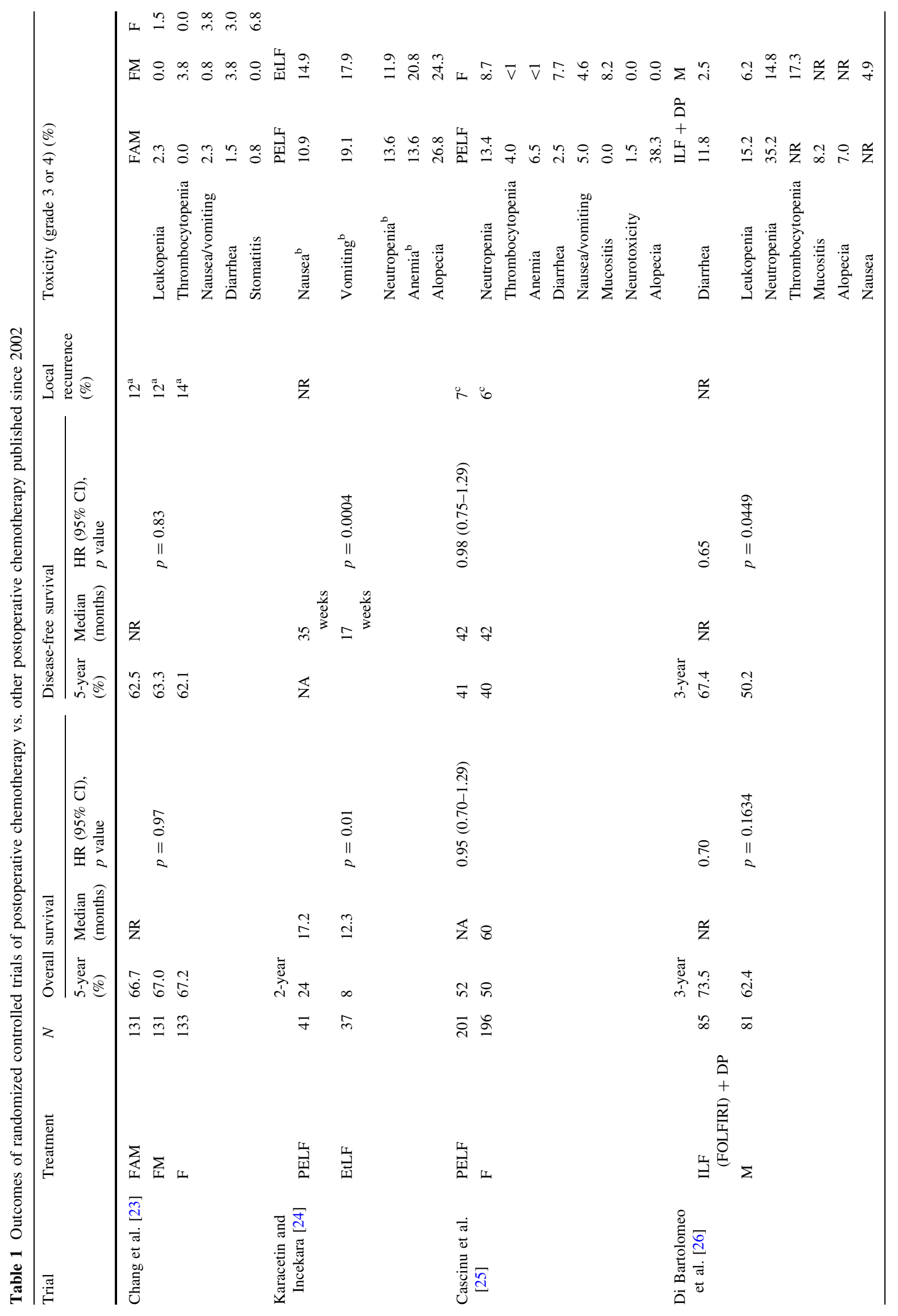




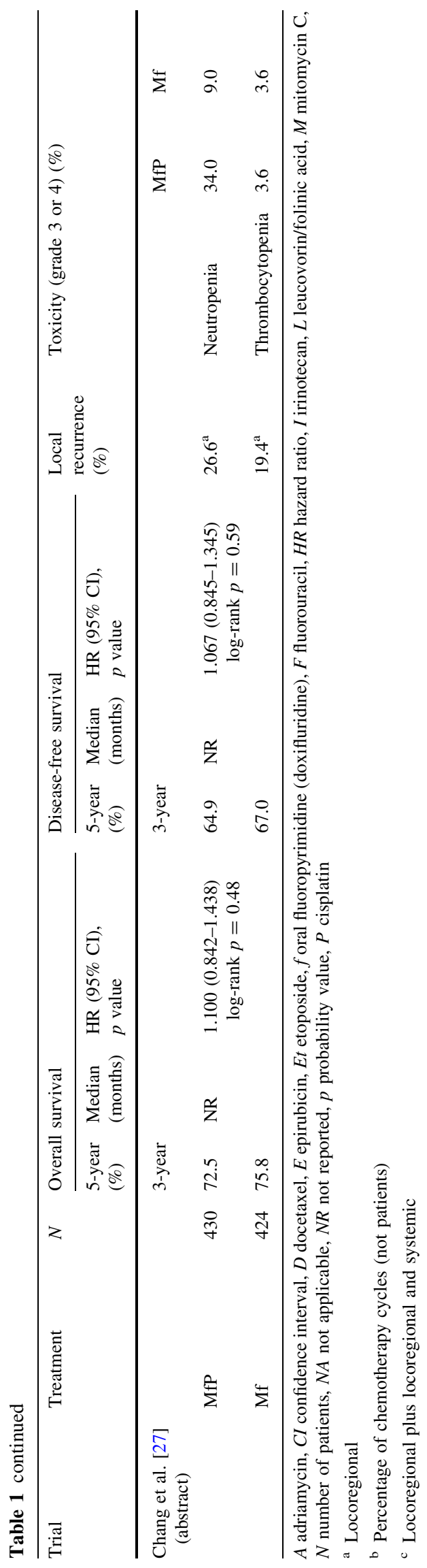

and 3-year OS or median and 3-year progression-free survival (PFS). This was not surprising, as the trial did not meet its accrual target and was, therefore, underpowered to detect a survival difference. The most common grade 3 and 4 toxicities reported, other than alopecia, were non-febrile neutropenia, febrile neutropenia, and diarrhea. However, the difference between the two arms was not statistically significant for any of these toxicities.

One meta-analysis of RCTs of postoperative CRT was identified [44]. Five RCTs were included, three of which compared postoperative CRT versus surgery alone, and two of which compared postoperative CRT versus postoperative chemotherapy. Meta-analysis of the five trials indicated no significant benefit for postoperative CRT over control in 3 -year mortality (OR $0.79,95 \%$ CI $0.59-1.05 ; p=0.10$ ); however, a meta-analysis of three trials that provided 5-year mortality data indicated a significant benefit for postoperative CRT over surgery (OR $0.45,95 \%$ CI $0.32-0.64$; $p<0.00001)$. No significant statistical heterogeneity between trials was reported. Fiorica et al. [44] reported that $52 \%$ of patients did not complete the CRT protocol as planned. Grades 3 and 4 hematologic and gastrointestinal toxicities, as well as mucositis, were significantly greater in the CRT arms compared to controls in this meta-analysis.

Two secondary analyses of the Southwest Oncology Group (SWOG)/Intergroup trial [49] were identified that also reported updated survival data [47, 48]. Updated results indicated a median survival of 36 months for patients who received postoperative CRT [5-FU/leucovorin (LV)] versus 27 months for patients who underwent surgery alone $(p=0.003)$. Relapse-free survival was 30 versus 19 months $(p<0.001)$, respectively. Further updates of the SWOG/ Intergroup trial were presented at ASCO in 2009 [48]. The abstract [48], based on 10 years of follow up, demonstrated continued benefit for the CRT group for both survival (HR $0.76 ; p=0.004)$ and DFS (HR 0.66; $p<0.001$ ). The presentation at ASCO of this abstract [48] was based on 11 years of follow up and demonstrated similar results for both OS (HR 0.76, 95\% CI 0.63-0.92; $p=0.005$ ) and DFS (HR 0.66, $95 \%$ CI $0.55-0.80 ; p<0.001)$. The original publication of the SWOG/Intergroup trial [49] reported that 33 and 54\% of patients in the CRT arm had grade 3 or higher hematologic and gastrointestinal toxicities, respectively.

Preoperative or perioperative chemotherapy

Two meta-analyses were identified that compared preoperative chemotherapy versus surgery alone [42, 43]. Both of these meta-analyses were available only in abstract form, providing only a limited amount of methodological information, and for this reason will not be discussed further. No meta-analyses were identified that compared perioperative chemotherapy versus surgery alone. Four RCT reports, 


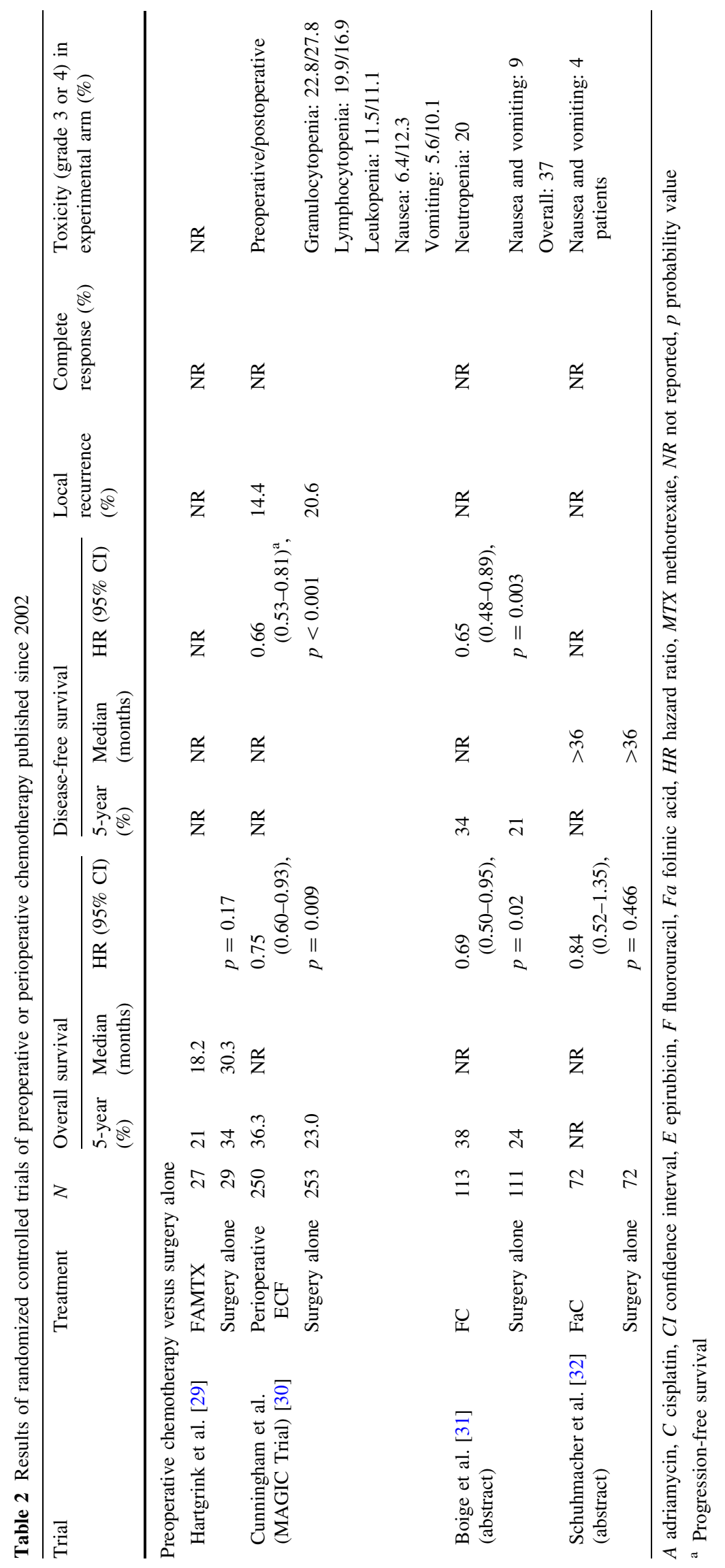


comparing preoperative or perioperative chemotherapy versus surgery alone, have been published since 2002 [29-32] (Table 2). One of the reports [29] presents long-term results of the Dutch trial by Songun et al. [50]. This trial was stopped after the accrual of 59 of a planned 450 patients, owing to the slow recruitment and poor interim results. No benefit for preoperative fluorouracil-doxorubicin-methotrexate (FAMTX) over surgery alone could be demonstrated. Another trial compared preoperative chemotherapy with folinic acid and cisplatin followed by surgery to surgery alone in patients with locally advanced adenocarcinoma of the stomach and cardia. This trial was stopped early owing to poor accrual. Only 144 of an expected 360 patients $(40 \%)$ were accrued during more than 4 years of the study. No survival benefit for the addition of preoperative chemotherapy was demonstrated [32]. Publication of the Fédération Nationale des Centres de Lutte Contre le Cancer (FNLCC) ACCORD07 [31] trial of 224 patients comparing preoperative 5-FU/cisplatin versus surgery alone in resectable gastric and lower esophageal cancer is available only in abstract form. A significant improvement in OS and DFS with preoperative 5-FU/cisplatin was reported (Table 2).

The MAGIC (Medical Research Council Adjuvant Gastric Infusional Chemotherapy) trial reported by Cunningham et al. [30] in 2006 is the largest trial incorporating preoperative therapy to date and the only randomized trial with a perioperative approach. A total of 503 patients were randomized to preoperative and postoperative epirubicin, cisplatin, fluorouracil (ECF) or surgery alone. Patients with adenocarcinoma of the stomach or lower-third of the esophagus who had stage II or higher (M0) disease or locally advanced inoperable disease were included. It should be noted that only $68 \%$ of patients underwent curative surgery, while the remaining patients had palliative surgery, no surgery, or surgery of unknown intent. Of the patients assigned to perioperative ECF, $41.6 \%$ completed all six cycles of chemotherapy, and $49.5 \%$ of the patients who completed preoperative ECF also completed postoperative therapy. A significant benefit for perioperative ECF was reported for OS and PFS (Table 2). Although results for patients with gastric and GEJ tumors were not reported separately from results for tumors of the lower esophagus, no heterogeneity of treatment effect according to disease site was demonstrated (interaction $p=0.25$ ).

Overall, preoperative and perioperative chemotherapy approaches resulted in greater hematologic toxicities, as well as greater incidences of nausea and vomiting, compared to surgery alone (Table 2).

Preoperative radiotherapy

Three published literature meta-analyses of trials comparing preoperative radiotherapy versus surgery alone were identified in the updated literature search [44-46], as well as a full publication of a trial by Skoropad et al. [33] included in abstract form in the original systematic review. The Skoropad trial [33] is included in the Fiorica et al. [44] meta-analysis and will not be discussed separately.

The meta-analysis by Fiorica et al. [44] included four RCTs of preoperative radiotherapy versus surgery alone, one of which combined preoperative radiotherapy with local hyperthermia. Results indicated a significant survival benefit for preoperative radiotherapy at both 3 years (OR $0.57,95 \%$ CI $0.43-0.76 ; p=0.0001$ ) and 5 years (OR $0.62,95 \% \mathrm{CI}$ $0.46-0.84 ; p<0.00001)$, and no significant statistical heterogeneity between trials was demonstrated. All patients in the studies of this meta-analysis were able to complete the preoperative radiation without dose reductions.

The meta-analysis by Valentini et al. [45] included studies of preoperative, postoperative, and intraoperative radiation, as well as radiation combined with chemotherapy all combined into one analysis. Because of this clinical heterogeneity, this meta-analysis will not be discussed further. The meta-analysis by Lu et al. [46] was available only in abstract form, and because only a limited amount of methodological information was provided, it will not be discussed further.

\section{Discussion}

Many trials and meta-analyses of trials have investigated the value of neoadjuvant and adjuvant treatment in gastric cancer. These efforts have produced conflicting results. The Gastrointestinal DSG decided that an update of EBS \#2-14, which was first developed in 2000 and updated in 2003, was justified, given the availability of new evidence that could change the recommendations made in the last version of this guidance document. As there may be biologic and etiologic differences in gastric cancer in the Asian population compared with the North American population, this guideline may not have the same relevance outside of the North American population.

\section{Postoperative chemotherapy}

The IPD meta-analysis by the GASTRIC group [41] demonstrated that there was a modest but significant survival advantage for postoperative chemotherapy, based on the 17 trials for which they could get IPD. This conclusion was maintained when a sensitivity analysis, which added in summary statistics for another 11 trials, was carried out.

Postoperative radiation

No trials solely comparing postoperative radiation therapy to surgery alone were identified in the updated literature search. 
Preoperative radiation

A published literature meta-analysis by Fiorica et al. [44] included four RCTs of preoperative radiotherapy versus surgery alone, one of which combined preoperative radiotherapy with local hyperthermia. Results indicated a significant survival benefit for preoperative radiotherapy at both 3 years (OR $0.57,95 \%$ CI $0.43-0.76$; $p=0.0001$ ) and 5 years (OR 0.62, 95\% CI 0.46-0.84; $p<0.00001$ ), and no significant statistical heterogeneity between trials was demonstrated.

A preoperative radiotherapy approach seems to provide a superior outcome with respect to 3- and 5-year OS. However, this treatment has not been taken up in the North American oncology community. There are four main reasons for this. First, the evidence for preoperative radiation originated predominantly from China and Russia. The generalizability of the results to Canadian/North American practice cannot be assumed. There was significant heterogeneity in the way the preoperative therapy was delivered. The radiotherapy used in three of the four studies used a large dose per fraction (20 Gy in 5 fractions) [51, 52], although one study did employ a standard 2-Gy dose per fraction (40 Gy in 20 fractions) [53]. Similarly, the target volume included for radiotherapy varied across the studies. These differences create challenges toward understanding how to implement these findings into practice. The magnitude of benefit as demonstrated through meta-analysis [44] is potentially smaller compared with a postoperative CRT approach [number needed to treat (NNT) for $\mathrm{RT}=10$ and for CRT $=6$ ] [44]. Finally, the high probability of both local and distant recurrence in gastric cancer has led to a preference towards strategies that incorporated both radiotherapy and chemotherapy.

The preference towards incorporating chemotherapy into adjuvant or neoadjuvant approaches is reflected by the fact that none of the clinical trials currently ongoing evaluate the use of preoperative radiation therapy alone, although the evaluation of preoperative CRT, perioperative chemotherapy, and postoperative CRT approaches continues to be actively pursued.

\section{Postoperative chemoradiation}

The meta-analysis by Fiorica et al. [44] of RCTs comparing postoperative chemoradiation to surgery alone did demonstrate a significant benefit with respect to 5-year mortality (OR 0.45 , 95\% CI 0.32-0.64; $p<0.00001$ ), although it is interesting to note that the results for 3-year mortality were not significant. This might be an indication that the 5-year results are spurious, though it is not possible to determine this. It should also be noted that one of the trials included in this meta-analysis is the Macdonald et al.
[49] SWOG/Intergroup trial. Updated survival data from this specific trial was identified [47] and indicated superior median survival for patients receiving postoperative chemoradiation over surgery alone (36 vs. 27 months; $p=0.003)$. Similarly, relapse-free survival was superior in the chemoradiation arm (30 vs. 19 months; $p<0.001)$. A further update of the SWOG/Intergroup trial demonstrated the robustness of these findings even after 11 years of follow up for both OS (HR 0.76, 95\% CI 0.63-0.92; $p=0.005$ ) and DFS (HR 0.66, 95\% CI $0.55-0.80$; $p<0.001$ ) [48].

In the Macdonald et al. SWOG/Intergroup trial [49], the protocol recommended that a D2 (more extensive) lymph node dissection be performed, but as many of the referrals to the trial occurred postoperatively, this could not be mandated. Upon final analysis, only $10 \%$ of patients had a D2 lymph node dissection, 36\% had a D1 lymph node dissection, and $54 \%$ had a D0 lymph node dissection (i.e., not all of the N1 nodes were removed). The lack of adequate lymph node dissection in over half of the SWOG/ Intergroup patients has led to criticism of the trial, with suggestions that the addition of adjuvant chemoradiation may be compensating for inadequate surgical resection [54, 55]. A subsequent observational trial, in which a D2 lymph node dissection was performed in the majority of patients, has upheld a survival benefit for adjuvant chemoradiation in patients who underwent more aggressive surgery [56]. In Korea, a prospective randomized phase 3 trial is being conducted in patients who have had a D2 dissection to further clarify the role of postoperative chemoradiation in this group of patients.

\section{Preoperative or perioperative chemotherapy}

The MAGIC trial [30] was a large trial of over 500 patients comparing perioperative chemotherapy (ECF) to surgery alone. This trial demonstrated significant improvement in 5-year OS (HR 0.75, 95\% CI 0.60-0.93; $p=0.009$ ) and 5-year PFS (HR 0.66, 95\% CI 0.53-0.81; $p<0.001$ ).

Considerations for choice of therapy

The Macdonald et al. [47-49] and the Cunningham et al./ MAGIC [30] trials have provided strong support for either a postoperative chemotherapy/radiotherapy approach to treatment or a perioperative approach, respectively.

The decision to initiate a perioperative chemotherapy approach versus the postoperative chemoradiation approach should be based on a number of patient- and tumor-specific factors and ideally should be made preoperatively.

Diagnostic laparoscopy is reasonable to consider prior to the initiation of perioperative chemotherapy to determine whether there is peritoneal spread of metastatic disease not 
detected on computerized tomography (CT) imaging, as this assessment may be less accurate following the administration of chemotherapy. While down-staging is not considered an indication for the MAGIC protocol, a perioperative approach does allow for assessment of biologic response to systemic chemotherapy, which may be important in clinical decision-making for patients with bulky tumors, or radiologically positive lymph nodes. Patients who are undergoing a total gastrectomy, as opposed to a sub-total gastrectomy, may have difficulty with nutrition postoperatively, especially when additional therapy is introduced, as described in the SWOG/Intergroup clinical trials [49]. A feeding tube should be considered for patients undergoing a total gastrectomy, with plans for postoperative therapies if there are doubts that the patient will be able to complete postoperative treatment because of poor caloric intake.

Some factors can be associated with increased or escalated risk of radiotherapy toxicities specifically. The anastomosis is typically included in the radiotherapy portal. For patients where the esophagogastric anastomosis or planned location is above the carina, the inclusion of this region that is required would predict for excessive lung and cardiac radiotherapy toxicities. The nodal regions and the blind loop post-resection are frequently immediately adjacent to the kidneys. For patients with borderline renal function, radiation is expected to be associated with an increased risk of chronic renal impairment. In these patients, depending on the severity of the renal dysfunction, consideration of the Cunningham approach [30], using chemotherapy alone, should be considered. There is a trade-off between the expected toxicity of radiation versus the toxicity of cisplatin. The relative toxicities of each approach need to be considered on a case-by-case-basis.

Similarly, there are factors that need to be considered for the use of perioperative chemotherapy. The presence of cardiac or significant renal dysfunction would contraindicate the use of epirubicin and cisplatin, respectively.

During the combined modality treatment of radiation and chemotherapy used during the Macdonald [49] protocol, some centers used a low-dose continuous 5-FU infusion or alternatively used oral capecitabine as a radiosensitizer. This would seem to be reasonable from a biologic perspective and is considered acceptable.

Clearly, all patients would benefit from a multidisciplinary care assessment prior to surgery in order to determine the best plan of care for each individual patient. Clinicians must tailor the decision to recommend postoperative CRT according to a patient's nutritional and performance status. Unless obviously contraindicated owing to poor performance status, all patients undergoing gastric surgery with curative intent should be considered for adjuncts to resection.
Technical considerations for radiation therapy

Many technical issues for the provision of radiation therapy have been introduced to refine and enhance the quality of the radiotherapy plan. The target volume is in the upper abdomen targeting the tumor bed and regional nodes, $2 \mathrm{~cm}$ beyond the proximal and distal margin of resection.

The extent of regional node irradiation is further modified based on the location of the primary tumor: for example, for T3 lesions in the proximal stomach, the medial left hemidiaphragm, was also included. The regional nodes were defined (based on the Japanese Research Society for Gastric Cancer) as perigastric, celiac, local para-aortic, splenic, hepatoduodenal or hepatic portal, and pancreaticodudenal. In addition, for GEJ tumors, the regional nodes included paracardial and para-esophageal lymph node beds, but excluded the pancreatic duodenal and splenic nodal beds. The latter were also excluded in antral tumors. Guidelines for more specific tailoring of nodal regions based on tumor location as well as $\mathrm{T}$ and $\mathrm{N}$ stage are provided by Tepper and Gunderson [57] and in a recent guideline for preoperative radiation treatments of the stomach published by the European Organisation for Research and Treatment of Cancer (EORTC) [58].

Strategies to incorporate internal organ motion into treatment planning allow for the further individualization of treatment plans. Respiratory motion can be incorporated through the use of four-dimensional computerized tomography (4-D CT) [59], and gastric volume variation can be reduced through instructions for 'standardized meals' prior to treatment planning and each treatment [60]. The use of renal perfusion scans allows for the refinement of radiotherapy beam geometry based on risk and organ function.

The use of conformal radiotherapy has generally superseded the techniques described in the original MacDonald study. Intensity-modulated radiation therapy (IMRT) techniques may provide further incremental benefit with lower doses to normal structures being achieved, although the optimal way of adopting this continues to be investigated [61, 62].

Permissible radiation dose limits for organs at risk (OAR) may affect the expected and observed long-term risks. More conservative parameters than those described in the original Macdonald [49] study have been recommended [58] and adopted into clinical practice.

\section{Conclusions}

Overall survival in patients with resectable gastric cancer is significantly improved with the use of either postoperative chemoradiation implementing the Macdonald protocol [47-49] or perioperative ECF implementing the MAGIC 
protocol [30]. The choice of which option to utilize should be based on individual patient factors affecting their ability to tolerate either the radiation used in the Macdonald protocol or the epirubicin/cisplatin used in the MAGIC protocol. If neither of these approaches is appropriate for a given patient, then postoperative chemotherapy is a reasonable alternative. All patients with resectable gastric cancer should undergo a multidisciplinary assessment to determine the best plan of care.

\section{Recommendations}

- Postoperative 5-fluorouracil (5-FU)-based chemoradiotherapy (CRT) based on the Macdonald approach [49] or perioperative epirubicin/cisplatin/5-FU (ECF) chemotherapy based on the Cunningham/Medical Research Council Adjuvant Gastric Infusional Chemotherapy (MAGIC) approach [30] are both acceptable standards of care in North America. Choice of treatment should be made on a case-by-case basis.

- Adjuvant chemotherapy is a reasonable option for those patients for whom the Macdonald [49] and MAGIC [30] protocols are contraindicated.

- Patients with resectable gastric cancer should undergo a pretreatment multidisciplinary assessment to determine the best plan of care. In addition to surgery, all patients should be considered for neoadjuvant and/or adjuvant therapy.

Acknowledgments The authors would like to thank the members of the GI DSG for their contributions to the development of this guidance document. This work was sponsored by Cancer Care Ontario and the Ontario Ministry of Health and Long-Term Care.

Conflict of interest GI DSG members involved in the development of the systematic review and clinical practice guideline were polled for potential conflicts of interest. All authors declared no conflicts of interest.

\section{References}

1. Parkin DM, Pisani P, Ferlay J. Global cancer statistics. CA Cancer J Clin. 1999;49(1):33-64.

2. Canadian Cancer Society's Steering Committee. Canadian Cancer Statistics 2009. Toronto: Canadian Cancer Society; 2009.

3. Middleton G, Cunningham D. Current options in the management of gastrointestinal cancer. Ann Oncol. 1995;6(Suppl 1):S17-26.

4. Hermans J, Bonenkamp JJ, Boon MC, Bunt AMG, Ohyama S, Sasako M, et al. Adjuvant therapy after curative resection for gastric cancer: meta-analysis of randomized trials. J Clin Oncol. 1993;11(8):1441-7.

5. Earle CC, Maroun JA. Adjuvant chemotherapy after curative resection for gastric cancer in non-Asian patients: revisiting a meta-analysis of randomised trials. Eur $\mathrm{J}$ Cancer. 1999;35(7):1059-64.
6. Mari E, Floriani I, Tinazzi A, Buda A, Belfiglio M, Valentini M, et al. Efficacy of adjuvant chemotherapy after curative resection for gastric cancer: a meta-analysis of published randomised trials. A study of the GISCAD (Gruppo Italiano per lo Studio dei Carcinomi dell'Apparato Digerente). Ann Oncol. 2000;11(7): 837-43.

7. Browman GP, Levine MN, Mohide EA, Hayward RSA, Pritchard KI, Gafni A, et al. The practice guidelines development cycle: a conceptual tool for practice guidelines development and implementation. J Clin Oncol. 1995;13(2):502-12.

8. Shea BJ, Bouter LM, Peterson J, Boers M, Andersson N, Ortiz Z, et al. External validation of a measurement tool to assess systematic reviews (AMSTAR). PLoS ONE. 2007;2(12):e1350. http:// www.plosone.org/article/info:doi/10.1371/journal.pone.0001350.

9. Oba K, Morita S, Tsuburaya A, Kodera Y, Kobayashi M, Sakamoto J. Efficacy of adjuvant chemotherapy using oral fluorinated pyrimidines for curatively resected gastric cancer: a metaanalysis of centrally randomized controlled clinical trials in Japan. J Chemother. 2006;18(3):311-7.

10. Sakamoto J, Morita S, Tsuburaya A, Kodera Y, Matsui T, Kobayashi $\mathrm{O}$, et al. Efficacy of adjuvant chemotherapy with oral fluorinated pyrimidines for patients with curatively resected gastric cancer. A meta-analysis of centrally randomized clinical trials. J Clin Oncol. 2005;23(16 Suppl):4022.

11. Sakamoto J, Tsuburaya A, Morita S, Matsui T, Oba K, Kodera Y, et al. Adjuvant chemotherapy with tegafur/uracil (UFT) for gastric cancer. A meta-analysis of centrally randomized clinical trials. J Clin Oncol. 2006;24(18 Suppl):4033.

12. Bajetta E, Buzzoni R, Mariani L, Beretta E, Bozzetti F, Bordogna $\mathrm{G}$, et al. Adjuvant chemotherapy in gastric cancer: 5-year results of a randomised study by the Italian Trials in Medical Oncology (ITMO) Group. Ann Oncol. 2002;13(2):299-307.

13. Nashimoto A, Nakajima T, Furukawa H, Kitamura M, Kinoshita $\mathrm{T}$, Yamamura $\mathrm{Y}$, et al. Randomized trial of adjuvant chemotherapy with mitomycin, fluorouracil, and cytosine arabinoside followed by oral fluorouracil in serosa-negative gastric cancer: Japan Clinical Oncology Group 9206-1. J Clin Oncol. 2003; 21(12):2282-7.

14. Chipponi J, Huguier M, Pezet D, Basso N, Hay J-M, Quandalle P, et al. Randomized trial of adjuvant chemotherapy after curative resection for gastric cancer. Am J Surg. 2004;187(3):440-5.

15. Popiela T, Kulig J, Czupryna A, Szczepanik AM, Zembala M. Efficiency of adjuvant immunochemotherapy following curative resection in patients with locally advanced gastric cancer. Gastric Cancer. 2004;7(4):240-5.

16. Bouche O, Ychou M, Burtin P, Bedenne L, Ducreux M, Lebreton $\mathrm{G}$, et al. Adjuvant chemotherapy with 5-fluorouracil and cisplatin compared with surgery alone for gastric cancer: 7-year results of the FFCD randomized phase III trial (8801). Ann Oncol. 2005;16(9):1488-97.

17. Nitti D, Wils J, Dos Santos JG, Fountzilas G, Conte PF, Sava C, et al. Randomized phase III trials of adjuvant FAMTX or FEMTX compared with surgery alone in resected gastric cancer. A combined analysis of the EORTC GI Group and the ICCG. Ann Oncol. 2006;17(2):262-9.

18. De Vita F, Giuliani F, Orditura M, Maiello E, Galizia G, Di Martino N, et al. Adjuvant chemotherapy with epirubicin, leucovorin, 5-fluorouracil and etoposide regimen in resected gastric cancer patients: a randomized phase III trial by the Gruppo Oncologico Italia Meridionale (GOIM 9602 Study). Ann Oncol. 2007;18(8):1354-8.

19. Nakajima T, Kinoshita T, Nashimoto A, Sairenji M, Yamaguchi $\mathrm{T}$, Sakamoto J, et al. Randomized controlled trial of adjuvant uracil-tegafur versus surgery alone for serosa-negative, locally advanced gastric cancer. Br J Surg. 2007;94(12):1468-76. 
20. Sakuramoto S, Sasako M, Yamaguchi T, Kinoshita T, Fujii M, Nashimoto A, et al. Adjuvant chemotherapy for gastric cancer with $\mathrm{S}-1$, an oral fluoropyrimidine. $\mathrm{N}$ Engl $\mathrm{J}$ Med. 2007;357(18):1810-20 (Erratum published in N Engl J Med. 2008 May 1;358(18):1977).

21. Di Costanzo F, Gasperoni S, Manzione L, Bisagni G, Labianca R, Bravi S, et al. Adjuvant chemotherapy in completely resected gastric cancer: a randomized phase III trial conducted by GOIRC. J Natl Cancer Inst. 2008;100(6):388-98.

22. Kulig J, Kolodziejczyk P, Sierzega M, Bobrzynski L, Jedys J, Popiela T, et al. Adjuvant chemotherapy with etoposide, adriamycin and cisplatin compared with surgery alone in the treatment of gastric cancer: a phase III randomized, multicenter, clinical trial. Oncology. 2010;78:54-61.

23. Chang HM, Jung KH, Kim TY, Kim WS, Yang HK, Lee KU, et al. A phase III randomized trial of 5-fluorouracil, doxorubicin, and mitomycin $\mathrm{C}$ versus 5 -fluorouracil and mitomycin $\mathrm{C}$ versus 5 -fluorouracil alone in curatively resected gastric cancer. Ann Oncol. 2002;13(11):1779-85.

24. Karacetin D, Incekara O. A randomized trial of 5-fluorouracil, leucovorin, cisplatin and epirubicin (PELF) versus 5-fluorouracil, leucovorin and etoposide (ELF) given as adjuvant chemotherapy to patients with resected advanced gastric adenocarcinomas. J BUON. 2004;9(3):263-7.

25. Cascinu S, Labianca R, Barone C, Santoro A, Carnaghi C, Cassano A, et al. Adjuvant treatment of high-risk, radically resected gastric cancer patients with 5-fluorouracil, leucovorin, cisplatin, and epidoxorubicin in a randomized controlled trial. $\mathrm{J}$ Natl Cancer Inst. 2007;99(8):601-7.

26. Di Bartolomeo M, Buzzoni R, Mariani L, Ferrario E, Katia D, Gevorgyan A, et al. Feasibility of sequential therapy with FOLFIRI followed by docetaxel/cisplatin in patients with radically resected gastric adenocarcinoma: a randomized phase III trial. Oncology. 2007;71(5-6):341-6.

27. Chang H, Kang Y, Min Y, Zang D, Kim G, Yang D, et al. A randomized phase III trial comparing mitomycin-C plus shortterm doxifluridine (Mf) versus mitomycin-C plus long-term doxifluridine plus cisplatin (MFP) after curative resection of advanced gastric cancer (AMC 0201) (NCT00296335). J Clin Oncol. 2008;26(15 Suppl):4531.

28. Bamias A, Karina M, Papkostas P, Kostopoulos I, Bobos M, Vourli G, et al. A randomized phase III study of adjuvant platinum/docetaxel chemotherapy with or without radiation therapy in patients with gastric cancer. Cancer Chemother Pharmacol. 2010;65:1009-21.

29. Hartgrink HH, van de Velde CJH, Putter H, Songun I, Tesselaar MET, Kranenbarg EK, et al. Neo-adjuvant chemotherapy for operable gastric cancer: long term results of the Dutch randomised FAMTX trial. Eur J Surg Oncol. 2004;30(6):643-9.

30. Cunningham D, Allum WH, Stenning SP, Thompson JN, Van de Velde $\mathrm{CJH}$, Nicolson $\mathrm{M}$, et al. Perioperative chemotherapy versus surgery alone for resectable gastroesophageal cancer. N Engl J Med. 2006;355(1):11-20.

31. Boige V, Pignon J, Saint-Aubert B, Lasser P, Conroy T, Bouche $\mathrm{O}$, et al. Final results of a randomized trial comparing preoperative 5-fluorouracil (F)/cisplatin (P) to surgery alone in adenocarcinoma of stomach and lower esophagus (ASLE): FNLCC ACCORD07-FFCD 9703. J Clin Oncol. 2007;25(18 Suppl):4510.

32. Schuhmacher C, Schlag P, Lordick F, Hohenberger W, Heise J, Haag C, et al. Neoadjuvant chemotherapy versus surgery alone for locally advanced adenocarcinoma of the stomach and cardia: randomized EORTC phase III trial \#40954. J Clin Oncol. 2009;25(15 Suppl):4510.

33. Skoropad V, Berdov B, Zagrebin V. Concentrated preoperative radiotherapy for resectable gastric cancer: 20 -years follow-up of a randomized trial. J Surg Oncol. 2002;80(2):72-8.
34. Hu J-K, Chen Z-X, Zhou Z-G, Zhang B, Tian J, Chen J-P, et al. Intravenous chemotherapy for resected gastric cancer: metaanalysis of randomized controlled trials. World J Gastroenterol. 2002;8(6):1023-8.

35. Janunger K-G, Hafstrom L, Glimelius B. Chemotherapy in gastric cancer: a review and updated meta-analysis. Eur J Surg. 2002;168(11):597-608.

36. Panzini I, Gianni L, Fattori PP, Tassinari D, Imola M, Fabbri P, et al. Adjuvant chemotherapy in gastric cancer: a meta-analysis of randomized trials and a comparison with previous meta-analyses. Tumori. 2002;88(1):21-7.

37. Hu J-K, Li C-M, Chen X-Z, Chen Z-X, Zhou Z-G, Zhang B, et al. The effectiveness of intravenous 5-fluorouracil-containing chemotherapy after curative resection for gastric carcinoma: a systematic review of published randomized controlled trials. J Chemother. 2007;19(4):359-75.

38. Zhao S-L, Fang J-Y. The role of postoperative adjuvant chemotherapy following curative resection for gastric cancer: a metaanalysis. Cancer Invest. 2008;26(3):317-25.

39. Liu TS, Wang Y, Chen SY, Sun YH. An updated meta-analysis of adjuvant chemotherapy after curative resection for gastric cancer. Eur J Surg Oncol. 2008;34(11):1208-16.

40. Sun P, Xiang JB, Chen ZY. Meta-analysis of adjuvant chemotherapy after radical surgery for advanced gastric cancer. Br J Surg. 2009;96(1):26-33.

41. GASTRIC (Global Advanced/Adjuvant Stomach Tumor Research International Collaboration) Group. Benefit of adjuvant chemotherapy for resectable gastric cancer: a meta-analysis. J Am Med Assoc. 2010;303:1729-37.

42. Li H, Zhu F, Cao Y, Zhai L, Lin T. Meta-analyses of randomized trials assessing the effect of neoadjuvant chemotherapy in locally advanced gastric cancer. American Society of Clinical Oncology Annual Meeting. J Clin Oncol. 2010;28(Abstract):4042.

43. Ronellenfitsch U, Schwarzbach M, Hofheinz R, Kienle P, Hohenberger $\mathrm{P}$, Jensen $\mathrm{K}$, et al. Meta-analysis of preoperative chemotherapy (CTX) versus primary surgery for locoregionally advanced adenocarcinoma of the stomach, gastroesophageal junction, and lower esophagus (GE adenocarcinoma). J Clin Oncol. 2010;28(15 Suppl):4022.

44. Fiorica F, Cartei F, Enea M, Licata A, Cabibbo G, Carau B, et al. The impact of radiotherapy on survival in resectable gastric carcinoma: a meta-analysis of literature data. Cancer Treat Rev. 2007;33(8):729-40.

45. Valentini V, Cellini F, Minsky BD, Mattiucci GC, Balducci M, D'Agostino G, et al. Survival after radiotherapy in gastric cancer: systematic review and meta-analysis. Radiother Oncol. 2009; 92(2):176-83.

46. Lu JJ, Liu T, Leong C, Tey J, Zhang Z. Survival benefits from preoperative radiation therapy in gastric carcinoma: a meta-analysis. Int J Radiat Oncol Biol Phys. 2009;75 Suppl 1:2172;S258.

47. Hundahl SA, Macdonald JS, Benedetti J, Southwest Oncology Group, Gastric Intergoup. Surgical treatment variation in a prospective, randomized trial of chemoradiotherapy in gastric cancer: the effect of undertreatment. Ann Surg Oncol. 2002; 9(3):278-86.

48. Macdonald JS, Benedetti J, Smalley S, Haller D, Hundahl S, Jessup J, et al. Chemoradiation of resected gastric cancer: a 10-year follow-up of the phase III trial INT0116 (SWOG 9008). J Clin Oncol. 2009;27(15 Suppl):4515.

49. Macdonald JS, Smalley SR, Benedetti J, Hundahl SA, Estes NC, Stemmermann GN, et al. Chemoradiotherapy after surgery compared with surgery alone for adenocarcinoma of the stomach or gastroesophageal junction. N Engl J Med. 2001;345(10): 725-30.

50. Songun I, Keizer HJ, Hermans J, Klementschitsch P, de Vries JE, Wils JA, et al. Chemotherapy for operable gastric cancer: results 
of the Dutch randomised FAMTX trial. The Dutch Gastric Cancer Group (DGCG). Eur J Cancer. 1999;35(4):558-62.

51. Skoropad VY, Berdov BA, Mardynski YS, et al. A prospective, randomized trial of pre-operative and intraoperative radiotherapy versus surgery alone in resectable gastric carcinoma. Eur J Surg Oncol. 2000;26:773-9.

52. Shchepotin IB, Evan SRT, Chorny V, et al. Intensive preoperative radiotherapy with local hypothermia for the treatment of gastric carcinoma. Surg Oncol. 1994;3:37-44.

53. Zhang ZX, Gu XZ, Yin WB, et al. Randomized clinical trial on the combination of preoperative irradiation and surgery in the treatment of adenocarcinoma of gastric cardia (AGC)-report on 370 patients. Int J Radiat Oncol Biol Phys. 1998;42:929-34.

54. Lordick F, Siewert JR. Recent advances in multimodal treatment for gastric cancer: a review. Gastric Cancer. 2005;8(2):78-85.

55. Dikken JL, Jansen EPM, Cats A, Bakker B, Hartgrink HH, Meershoek-Klein Kranenbarg E, et al. Impact of the extent of surgery and postoperative chemoradiotherapy on recurrence patterns in gastric cancer. J Clin Oncol. 2010;26(15 Suppl):9654.

56. Kim S, Lim DH, Lee J, Kang WK, MacDonald JS, Park CH, et al. An observational study suggesting clinical benefit for adjuvant postoperative chemoradiation in a population of over 500 cases after gastric resection with D2 nodal dissection for adenocarcinoma of the stomach. Int J Radiat Oncol Biol Phys. 2005; 63(5):1279-85.
57. Tepper JE, Gunderson LL. Radiation treatment parameters in the adjuvant postoperative therapy of gastric cancer. Semin Radiat Oncol. 2002;12(2):187-95.

58. Matzinger O, Gerber E, Bernstein Z, Maingon P, Haustermans K, Bosset JF, et al. EORTC-ROG expert opinion: radiotherapy volume and treatment guidelines for neoadjuvant radiation of adenocarcinomas of the gastroesophageal junction and the stomach. Radiother Oncol. 2009;92(2):164-75.

59. Zhao K-L, Liao Z, Bucci MK, Komaki R, Cox JD, Yu ZH, et al. Evaluation of respiratory-induced target motion for esophageal tumors at the gastroesophageal junction. Radiother Oncol. 2007;84(3):283-9.

60. Watanabe M, Isobe K, Takisima H, Uno T, Ueno N, Kawakami $\mathrm{H}$, et al. Intrafractional gastric motion and interfractional stomach deformity during radiation therapy. Radiother Oncol. 2008;87(3): 425-31.

61. Alani S, Soyfer V, Strauss N, Schifter D, Corn BW. Limited advantages of intensity-modulated radiotherapy over 3D conformal radiation therapy in the adjuvant management of gastric cancer. Int J Radiat Oncol Biol Phys. 2009;74(2):562-6.

62. van der Geld YG, Senan S, van Sornsen de Koste JR, Verbakel WFAR, Slotman BJ, Lagerwaard FJ. A four-dimensional CTbased evaluation of techniques for gastric irradiation. Int J Radiat Oncol Biol Phys. 2007;69(3):903-9. 